Article

\title{
Nonexistence of Positive Solutions for Quasilinear Equations with Decaying Potentials
}

\author{
Ohsang Kwon \\ Department of Mathematics, Chungbuk National University, Chungdae-ro 1, Seowon-gu, Cheongju, \\ Chungbuk 362-763, Korea; ohsangkwon@chungbuk.ac.kr
}

Received: 7 February 2020; Accepted: 12 March 2020; Published: 16 March 2020

\begin{abstract}
In this paper, we consider a quasilinear Schrödinger equation, which arises from the study of the superfluid film equation in plasma physics. Our main goal is to find the growth condition for nonlinear term and decaying condition for the potential, which guarantee the nonexistence of positive solutions.
\end{abstract}

Keywords: decaying potential; quasilinear equation; nonexistence

\section{Introduction}

In this paper, we consider the following quasilinear Schrödinger equation:

$$
\left\{\begin{array}{lr}
i \varepsilon \phi_{t}+\varepsilon^{2} \Delta \phi-W(x) \phi+\varepsilon^{2} \phi l^{\prime}\left(|\phi|^{2}\right) \Delta l\left(|\phi|^{2}\right)+\rho\left(|\phi|^{2}\right) \phi=0 & \text { in }(0, \infty) \times \mathbb{R}^{N} \\
\phi(0, x)=a_{0}(x) & \text { in } \mathbb{R}^{N}, N \geq 1
\end{array}\right.
$$

where $\phi: \mathbb{R} \times \mathbb{R}^{N} \rightarrow \mathbb{C}$ is a complex valued function, $W: \mathbb{R}^{N} \rightarrow \mathbb{R}$ is a given potential, $l, \rho:[0, \infty) \rightarrow \mathbb{R}$ are given functions, and $i$ is the imaginary unit.

Depending on the structure of the quasilinear term $l\left(|\phi|^{2}\right)$ in (1), the above equation describes various physical phenomena. For example, the self-channeling of a high-power ultrashort laser in matter is related to the type $l(s)=\sqrt{1+s}$ (see References [1-5]), and the type $l(s)=\sqrt{s}$ was considered for the study of dissipative quantum mechanics in Reference [6]. We also refer to References [7,8] for the study of plasma physics and fluid mechanics, Reference [9] for Heisenberg ferromagnetic and magnon, and Reference [10] for the condensed matter theory.

In this paper, we are mainly interested in the type $l(s)=s$, which arises from the superfluid film equation in plasma physics and self-trapped electrons in quadratic or hexagonal lattices (see References [11-15]). We consider the standing wave solution $u(x)$, that is, solution of the form $\phi(t, x)=e^{-i E t} u(x)$, where $E \in \mathbb{R}$. Then, (1) can be rewritten by $u(x)$ as follows:

$$
\varepsilon^{2} \Delta u+\varepsilon^{2} \Delta\left(u^{2}\right) u-V(x) u+f(u)=0, \text { in } \mathbb{R}^{N},
$$

where $V(x)=W(x)-E$ and $f(u)=\rho\left(u^{2}, x\right) u$.

When $f(u)=u^{p}$, Cheng and Wei in Reference [16] proved the existence of positive solution with slow fast decaying depending on a range of $p$ by using a perturbation method, and the authors of References [17-19] obtained some existence results by applying the constrained minimization argument. Under Ambrosetti-Rabinowitz type condition on $f$, Aires and Souto in Reference [20] showed the existence of positive solutions for (2) with the potential vanishing at infinity, and Severo and Carvalho in Reference [21], proved the existence of solutions with the potential, which has the singularity at the origin and possesses unbounded or vanishing condition at infinity. Liu et al in 
Reference [17] studied the energy functional after a change of variables by using Orlicz space. We also refer to Reference [22] for the existence result on a smooth bounded domain.

On the other hand, so far, there are only few results on a nonexistence of solutions. Chen et al in Reference [23] derived the nonexitence of solutions under the linear growth condition for $f$ and nonvanishing condition for $V$ at infinity. Moreover, Xue et al in Reference [24] obtained a nonexistence result by using the Pohozaev identity. These results deal with either almost linear or critical growth, whereas we consider subcritical growth in this paper.

Our main goal is to study the nonexistence of positive solution for the following equation with the potential $V(x)$ decaying at infinity:

$$
\left\{\begin{array}{l}
\varepsilon^{2} \Delta u+\varepsilon^{2} \Delta\left(u^{2}\right) u-V(x) u+u^{p}=0, \text { in } \mathbb{R}^{N}, \\
\lim _{|x| \rightarrow \infty} u(x)=0 .
\end{array}\right.
$$

Before stating our results precisely, let us first review the following classical elliptic equation,

$$
\varepsilon^{2} \Delta u-V(x) u+u^{p}=0, \text { in } \mathbb{R}^{N} .
$$

Bae and Byeon in Reference [25] found almost optimal threshold on the decaying condition of $V(x)$ at infinity between existence and nonexistence of positive solutions of (4). By the decaying condition, the Equation (3) has the threshold for the existence and nonexistence of positive solution. So, it is an interesting question to find some condition of threshold for our equation. In this paper, we obtain a partial answer for the condition of decaying rate which guarantees the nonexistence of positive solution. Compared with the elliptic Equation (4), the main difficulty for our main Equation (3) is how to deal with the quasilinear term $\varepsilon^{2} \Delta\left(u^{2}\right) u$. In order to avoid this difficulty, a transformation $g$ by a change of variables (see (6) for the setting of $g$ ) has been introduced (for example, see Reference [17]). However, the terms coming from the transformation $g$ cause another obstacle in the analysis, so our strategy to overcome this difficulty is to apply the inverse transformation with the spherical average. After these transformations, we could regard (3) as a perturbation of the nonlinear Equation (4).

Let $\mathfrak{N}=+\infty$ for $N=1,2$ and $\mathfrak{N}=\frac{N}{N-2}$ for $N \geq 3$.

We introduce the following conditions for the potential function $V$ :

(V1) $V \in \mathrm{C}\left(\mathbb{R}^{N}, \mathbb{R}^{+}\right)$;

(V2) if $1<p<\mathfrak{N}$, then $V(x)|x|^{2} \leq \frac{2}{p-1}\left[\frac{2}{p-1}+2-N\right]$ for sufficiently large $|x|$, and if $N \geq 3$ and $p=\frac{N}{N-2}$, then there exists some constant $\delta>0$ such that

$$
V(x)|x|^{2} \ln |x| \leq \frac{(N-2)^{2}}{2}+\frac{N(N-2)}{4 \ln |x|}-\frac{\delta}{\ln (\ln |x|)} \text { for sufficiently large }|x| ;
$$

(V3) if $1<p<\mathfrak{N}$, then $\lim _{|x| \rightarrow \infty} V(x)|x|^{2}=0$,

and if $N \geq 3$ and $p=\frac{N}{N-2}$, then $\lim _{|x| \rightarrow \infty} V(x)|x|^{2} \ln |x|=0$.

We state our main theorem as follows:

Theorem 1. Let $p>1$ when $N=1,2$ and $1<p \leq \frac{N}{N-2}$ when $N \geq 3$. We assume that the hypotheses (V1) and (V2) hold. Then, (3) has no positive $C^{2}$ solutions for $\varepsilon=1$.

Due to the scaling, we can extend Theorem 1 for any $\varepsilon>0$ as the follows:

Theorem 2. Let $p>1$ when $N=1,2$ and $1<p \leq \frac{N}{N-2}$ when $N \geq 3$. We assume that the hypotheses (V1) and (V3) hold. Then, (3) has no positive $C^{2}$ solutions for any $\varepsilon>0$. 
Moreover, we also consider the following equation with the nonvanishing boundary condition:

$$
\left\{\begin{array}{l}
\Delta u+\Delta\left(u^{2}\right) u-V(x) u+u^{p}=0 \text { in } \mathbb{R}^{N}, \\
\liminf |x| \rightarrow \infty \\
u(x)>0 .
\end{array}\right.
$$

For the above quasilinear Equation (5), we have the following result:

Theorem 3. Let $p>1$ when $N=1,2$ and $1<p \leq \frac{N}{N-2}$ when $N \geq 3$. We assume that the hypotheses (V1) and (V2) hold. Then, (5) has no positive $C^{2}$ solutions for any $\varepsilon>0$.

Remark 1. If $p$ is large enough, then (3) has infinitely many positive solutions even if $V$ decays faster than condition (V2) (see Reference [16]).

\section{Proof of Theorem 1}

We consider a change of variables $w=g^{-1}(u)$, where $u$ is a positive solution of (3) and $g$ is a smooth function satisfying

$$
\left\{\begin{array}{l}
g^{\prime}(t)=\left(1+2 g^{2}(t)\right)^{-\frac{1}{2}} \quad \text { if } t>0 \\
g(0)=0 \\
g(t)=-g(-t) \text { if } t<0
\end{array}\right.
$$

Then $w$ satisfies

$$
\Delta w+\frac{1}{\sqrt{1+2 g^{2}(w)}}\left\{-V(x) g(w)+g^{p}(w)\right\}=0 \text { in } \mathbb{R}^{N} .
$$

By using (6), we get

$$
\begin{aligned}
\frac{d^{2}}{d t^{2}}\left(\frac{g(t)}{\sqrt{1+2 g^{2}(t)}}\right) & =\frac{d}{d t}\left(\frac{g^{\prime}}{\sqrt{1+2 g^{2}}}-\frac{2 g^{2} g^{\prime}}{\left(1+2 g^{2}\right)^{\frac{3}{2}}}\right)=\frac{d}{d t}\left(\frac{1}{\left(1+2 g^{2}\right)^{2}}\right) \\
& =-8 g(t)\left(1+2 g^{2}(t)\right)^{-\frac{7}{2}}<0, \quad \text { for any } t>0 .
\end{aligned}
$$

Therefore, $\frac{g}{\sqrt{1+2 g^{2}}}$ is concave. We also get the following identity:

$$
\frac{d^{2}}{d t^{2}}\left(\frac{g^{p}}{\sqrt{1+2 g^{2}}}\right)=g^{p-2}\left(1+2 g^{2}\right)^{-\frac{7}{2}}\left\{4(p-1)(p-3) g^{4}+2\left(2 p^{2}-5 p-1\right) g^{2}+p(p-1)\right\} .
$$

Suppose that there exists a positive solution $w$ of (7). Then, $w$ is a supersolution of (7) in a complement of a large ball, in other words,

$$
\Delta w+\frac{1}{\sqrt{1+2 g^{2}(w)}}\left\{-V(x) g(w)+g^{p}(w)\right\} \leq 0,
$$

in the exterior domain $|x| \geq R$, where $R>0$ is sufficiently large. Therefore, it is enough to show that there is no supersolution of (7). Since 0 is the unique zero of $g$ and $\lim _{|x| \rightarrow \infty} w(x)=0$, we see that $\lim _{|x| \rightarrow \infty} g(w(x))=0$. Together with (8), we note that if $p>1$, then

$$
\frac{d^{2}}{d w^{2}}\left(\frac{g^{p}(w)}{\sqrt{1+2 g^{2}(w)}}\right)=g^{p-2}\left(1+2 g^{2}\right)^{-\frac{7}{2}}\{p(p-1)+o(1)\}>0 \text { as }|x| \rightarrow \infty .
$$


So the function $\frac{g^{p}(w)}{\sqrt{1+2 g^{2}(w)}}$ is convex with respect to $w(x)$ if $p>1$ and $|x|$ is sufficiently large. Let $\bar{w}$ be the spherical average of $w$. Namely, we set

$$
\bar{w}(r)=\frac{1}{\left|\partial B_{r}\right|} \int_{\partial B_{r}} w(x) d S,
$$

where $\partial B_{r}=\left\{x \in \mathbb{R}^{N}|| x \mid=r\right\}$. The following identity is well known from the proof of ([26], Theorem 2.1)

$$
\frac{d \bar{w}}{d r}=\left(\frac{1}{\sigma_{n} r^{N-1}} \int_{\partial B_{r}} w d S\right)^{\prime}=\frac{1}{\left|\partial B_{r}\right|} \int_{\partial B_{r}} \frac{\partial w}{\partial v} d S=\frac{1}{\left|\partial B_{r}\right|} \int_{B_{r}} \Delta w d x,
$$

where $\sigma_{n}$ is the area of unit sphere in $\mathbb{R}^{N}$ and $v$ is the outer normal unit vector on $\partial B_{r}$. Since $\bar{w}$ is radially symmetric, we see that

$$
\begin{aligned}
\Delta \bar{w} & =\frac{d^{2} \bar{w}}{d r^{2}}+\frac{N-1}{r} \frac{d \bar{w}}{d r}=\frac{1}{\sigma_{n} r^{N-1}} \frac{d}{d r}\left(\sigma_{n} r^{N-1} \frac{d \bar{w}}{d r}\right) \\
& =\frac{1}{\left|\partial B_{r}\right|} \frac{d}{d r}\left(\int_{B_{r}} \Delta w d x\right)=\frac{1}{\left|\partial B_{r}\right|} \int_{\partial B_{r}} \Delta w d S .
\end{aligned}
$$

Since $w$ is a supersolution of (7), we get that

$$
\Delta \bar{w}=\frac{1}{\left|\partial B_{r}\right|} \int_{\partial B_{r}} \Delta w d S \leq \frac{1}{\left|\partial B_{r}\right|} \int_{\partial B_{r}} \frac{V(x) g(w)}{\sqrt{1+2 g^{2}(w)}}-\frac{g^{p}(w)}{\sqrt{1+2 g^{2}(w)}} d S .
$$

By using the Jensen's inequality, we obtain that

$$
\Delta \bar{w} \leq \frac{1}{\left|\partial B_{r}\right|} \tilde{V}(r) \int_{\partial B_{r}} \frac{g(w)}{\sqrt{1+2 g^{2}(w)}} d S-\frac{1}{\left|\partial B_{r}\right|} \int_{\partial B_{r}} \frac{g^{p}(w)}{\sqrt{1+2 g^{2}(w)}} d S,
$$

where $\tilde{V}(r)=\max _{|x|=r} V(x)$.

To simplify (10), let $\bar{u}(r)=g(\bar{w}(r))$. We see that

$$
\bar{w}^{\prime}=\sqrt{1+2 \bar{u}^{2}} \bar{u}^{\prime} \quad \text { and } \quad \bar{w}^{\prime \prime}=\sqrt{1+2 \bar{u}^{2}} \bar{u}^{\prime \prime}+\frac{2 \bar{u}\left(\bar{u}^{\prime}\right)^{2}}{\sqrt{1+2 \bar{u}^{2}}} .
$$

Then, (10) can be rewritten by

$$
\bar{u}^{\prime \prime}+\frac{N-1}{r} \bar{u}^{\prime}+\frac{1}{1+2 \bar{u}^{2}}\left(2 \bar{u}\left(\bar{u}^{\prime}\right)^{2}-\tilde{V} \bar{u}+\bar{u}^{p}\right) \leq 0 .
$$

Since $2 \bar{u}\left(\bar{u}^{\prime}\right)^{2}$ is nonnegative, it follows that

$$
\bar{u}^{\prime \prime}+\frac{N-1}{r} \bar{u}^{\prime}-\frac{\tilde{V}}{1+2 \bar{u}^{2}} \bar{u}+\frac{1}{1+2 \bar{u}^{2}} \bar{u}^{p} \leq 0 .
$$

Then, since $\bar{u}$ converges to 0 at infinity, (11) can be regarded as a perturbation of supersolution for (4) in the exterior domain $|x| \geq R$. So, we can apply the arguments in Bae-Byeon [25].

We define $v(t) \equiv r^{m} \bar{u}(r)$ with $t=\ln r$ and $m=\frac{2}{p-1}$. Then, (11) can be rewritten by

$$
v^{\prime \prime}+(N-2-2 m) v^{\prime}+\left\{m(2+m-N)-\frac{\tilde{V}\left(e^{t}\right) e^{2 t}}{1+2 e^{-2 m t} v^{2}}\right\} v+\frac{1}{1+2 e^{-2 m t} v^{2}} v^{p} \leq 0 .
$$

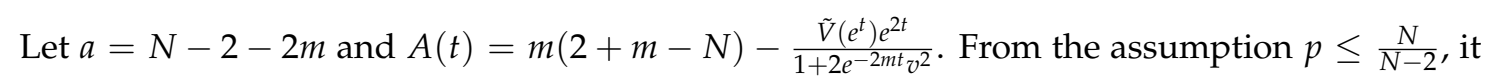
follows that $a<0$ and $m(2+m-N) \geq 0$. 
Firstly, we consider the case $m(2+m-N)>0$, that is, either $p<\frac{N}{N-2}$ or $N=1,2$. From the assumption (V2), there exists a constant $T>0$ satisfying $A(t)>0$ for $t \geq T$.

Multiplying $e^{a t}$ on both sides and integrating over $\left[t_{1}, t_{2}\right)$ for $T \leq t_{1} \leq t_{2}$, we obtain

$$
\begin{aligned}
v^{\prime}\left(t_{2}\right) & \leq e^{-a\left(t_{2}-t_{1}\right)} v^{\prime}\left(t_{1}\right)-e^{-a t_{2}} \int_{t_{1}}^{t_{2}}\left(A+\frac{v^{p-1}}{1+2 e^{-2 m t} v^{2}}\right) v e^{a s} d s \\
& \leq e^{-a\left(t_{2}-t_{1}\right)} v^{\prime}\left(t_{1}\right) .
\end{aligned}
$$

Since $a<0$, we note that if there exists a large constant $T_{1}$ satisfying $v^{\prime}\left(T_{1}\right)<0$, then $v$ has a finite zero. This is a contradiction. Therefore, $v(t)$ is non-decreasing for large $t$.

Assume that $v$ is bounded, so that $\lim _{t \rightarrow+\infty} v(t)=v_{\infty}>0$. Then, we claim that there exists a sequence $\left\{t_{j}\right\}$ such that $\lim _{j \rightarrow \infty} t_{j}=\infty, v^{\prime}\left(t_{j}\right), v^{\prime \prime}\left(t_{j}\right) \rightarrow 0$ as $j \rightarrow \infty$. To prove our claim, we need to consider the following three cases;

(i) $v^{\prime \prime}(t)$ is nonnegative for large $t$,

(ii) $v^{\prime \prime}(t)$ is nonpositive for large $t$,

(iii) $v^{\prime \prime}(t)$ has infinitely many sign-changing points.

Case (i): If there is a constant $t_{1}$ such that $v^{\prime \prime}(t) \geq 0$ for $t \geq t_{1}$, then $v^{\prime}(t)$ is nondecreasing for $t \geq t_{1}$. We also recall that there is a constant $t_{2}>0$ such that

$$
v^{\prime}(t) \geq 0 \text { for } t \geq t_{2}
$$

If there is a constant $t_{3} \geq \max \left\{t_{1}, t_{2}\right\}$ such that $v^{\prime}\left(t_{3}\right)>0$, then $v^{\prime}(t) \geq v^{\prime}\left(t_{3}\right)>0$ for $t \geq t_{3}$, which implies that $v(t)$ is unbounded. This contradicts the assumption $\lim _{t \rightarrow \infty} v(t)=v_{\infty}$. Therefore, the case (i) implies that $v^{\prime}(t)=v^{\prime \prime}(t)=0$ for $t \geq \max \left\{t_{1}, t_{2}\right\}$. Then, our claim holds in this case.

Case (ii): If there is a constant $t_{1}^{*}$ such that $v^{\prime \prime}(t) \leq 0$ for $t \geq t_{1}^{*}$, then $v^{\prime}(t)$ is nonincreasing for $t \geq t_{1}^{*}$. Let $t_{2}^{*}:=\max \left\{t_{1}^{*}, t_{2}\right\}$. In view of the nonincreasing property of $v^{\prime}(t)$ and (13), we see that if $t \geq t_{2}^{*}$, then

$$
\frac{v(t)-v\left(t_{2}^{*}\right)}{t-t_{2}^{*}}=\frac{\int_{t_{2}^{*}}^{t} v^{\prime}(s) d s}{t-t_{2} *} \geq v^{\prime}(t) \geq 0 .
$$

By letting $t \rightarrow \infty$, we get that $\lim _{t \rightarrow \infty} v^{\prime}(t)=0$ from the boundedness of $v(t)$. Moreover, since $v^{\prime}(t)$ is bounded below and nonincreasing for large $t>0$, we can find a sequence $\left\{t_{j}\right\}$ satisfying $\lim _{j \rightarrow \infty} t_{j}=\infty$ and $\lim _{j \rightarrow \infty} v^{\prime \prime}\left(t_{j}\right)=0$. Then, our claim holds in this case.

Case (iii): We can find a sequence $\left\{\tilde{t}_{j}\right\}$ satisfying $v^{\prime \prime}\left(\tilde{t}_{j}\right)=0$ and $v^{\prime \prime}(t)$ is nondecreasing around $\tilde{t}_{j}$. This implies that $\tilde{t}_{j}$ is a local minimum point of $v^{\prime}(t)$. Since $v(t)$ is bounded and $v^{\prime}(t)$ is nonnegative for large $t$, we see that $\lim _{j \rightarrow \infty} v^{\prime}\left(\tilde{t}_{j}\right)=0$. Then, our claim holds in this case.

From (12), we see that

$$
0<\frac{v_{\infty}^{p}}{2}<v^{\prime \prime}\left(t_{j}\right)+(N-2-2 m) v^{\prime}\left(t_{j}\right)+A\left(t_{j}\right) v\left(t_{j}\right)+\frac{1}{1+2 e^{-2 m t_{j}} v^{2}\left(t_{j}\right)} v^{p}\left(t_{j}\right) \leq 0 .
$$

This is a contradiction. Therefore, $v$ is unbounded and non-decreasing for large $t$.

Define $X(t)=e^{\frac{a t}{2}} v(t)$. Then, (12) can be rewritten by

$$
X^{\prime \prime}(t)+\left\{-\frac{(N-2)^{2}}{4}-\frac{\tilde{V}\left(e^{t}\right) e^{2 t}}{1+2 e^{-2 m t} v^{2}(t)}+\frac{1}{1+2 e^{-2 m t} v^{2}(t)} v^{p-1}(t)\right\} X(t) \leq 0 .
$$


Multiplying by $\sin t$ and integrating over $[2 k \pi,(2 k+1) \pi]$ for large integer $k$, we obtain

$$
\begin{aligned}
& \left.\int_{2 k \pi}^{(2 k+1) \pi}\left\{\frac{1}{1+2 e^{-2 m t} v^{2}} v^{p-1}-\left(\frac{N-2}{2}\right)^{2}-\frac{\tilde{V} e^{2 t}}{1+2 e^{-2 m t} v^{2}}-1\right)\right\} X \sin t \\
& \leq-X(2 k \pi)-X((2 k+1) \pi) \leq 0,
\end{aligned}
$$

which is a contradiction since the left hand side is positive for a sufficiently large integer $k$.

Secondly, we consider the case $m(2+m-N)=0$, that is, $p=\frac{N}{N-2}$ and $N \geq 3$.

Let $\varphi(r)=r^{2-N}(\ln r)^{\frac{2-N}{2}}(\ln (\ln r))^{\alpha}$ for $0<\alpha<\frac{\delta}{N-2}$. Then, we can check that

$$
\begin{aligned}
& \Delta \varphi(r)-\tilde{V}(r) \varphi(r) \\
& \geq r^{-N}(\ln r)^{\frac{-N-2}{2}}(\ln (\ln r))^{\alpha-1}\left[\{\delta-\alpha(N-2)\} \ln r-\alpha(N-1)+\frac{\alpha(\alpha-1)}{\ln (\ln r)}\right] \geq 0,
\end{aligned}
$$

for sufficiently large $r>0$. From comparison principle, (11) yields that there exist positive constants $C, R_{0}$ satisfying $\bar{u}(r) \geq C \varphi(r)$ if $r \geq R_{0}$. We also see that

$$
v^{\prime \prime}(t)+(2-N) v^{\prime}(t)-\frac{\tilde{V}\left(e^{t}\right) e^{2 t}}{1+2 e^{-2 m t} v^{2}} v(t)+\frac{1}{1+2 e^{-2 m t} v^{2}} v^{p}(t) \leq 0,
$$

where $t=\ln r$ and $v(t)=r^{N-2} \bar{u}(r)$. Since $\bar{u}(r) \geq C \varphi(r)$ for some $C>0$ and large $r,-\frac{\tilde{V}\left(e^{t}\right) e^{2 t}}{1+2 e^{-2 m t} v^{2}} v(t)+$ $\frac{1}{1+2 e^{-2 m t} v^{2}} v^{p}(t) \geq \frac{1}{2} v^{p}(t)$ for large $t$. By the similar argument for the case $m(2+m-N)>0$, we can show that $v$ is nondecreasing and derive a contradiction.

This completes the proof of Theorem 1.

Proof for Theorem 2. By using the scaling $y=\varepsilon^{-1} x$, (3) can be rewritten by

$$
\Delta u+\Delta\left(u^{2}\right) u=\left(V(\varepsilon y) u-u^{p}\right) .
$$

Then, for fixed $\varepsilon>0$, the assumption (V3) yields the assumption (V2). Therefore, Theorem 2 immediately follows from Theorem 1 .

Proof for Theorem 3. Let $u$ be a positive solution of (5) and $w=g^{-1}(u)$. From the condition (V2) and

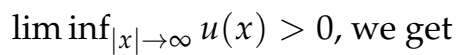

$$
-V(x) g(w)+g^{p}(w) \geq \frac{1}{2} g^{p}(w) \geq C>0,
$$

for some constant $C>0$ and sufficiently large $|x|$. By using the similar arguments in the proof of Theorem 1 , we can derive a contradiction.

Funding: NRF-2016R1C1B2014942.

Acknowledgments: The author was supported by Young Researcher Program through the National Research Foundation of Korea (NRF) funded by the Ministry of Education (no. NRF-2016R1C1B2014942).

Conflicts of Interest: The author declares no conflict of interest.

\section{References}

1. Borovskii, A.V.; Galkin, A.L. Dynamical modulation of an ultrashort high-intensity laser pulse in matter. JETP 1993, 77, 562-573.

2. De Bouard, A.; Hayashi, N.; Saut, J.C. Global existence of small solutions to a relativistic nonlinear Schrödinger equation. Commun. Math. Phys. 1997, 189, 73-105. [CrossRef] 
3. Brandi, H.S.; Manus, C.; Mainfray, G.; Lehner, T.; Bonnaud, G. Relativistic and ponderomotive self-focusing of a laser beam in a radially inhomogeneous plasma. Phys. Fluids B 1993, 5, 3539-3550. [CrossRef]

4. Chen, X.L.; Sudan, R.N. Necessary and sufficient conditions for self-focusing of short ultraintense laser pulse. Phys. Rev. Lett. 1993, 70, 2082-2085. [CrossRef]

5. Ritchie, B. Relativistic self-focusing and channel formation in laser-plasma interactions. Phys. Rev. E 1994, 50, 687-689. [CrossRef]

6. Hasse, R.W. A general method for the solution of nonlinear soliton and kink Schrödinger equations. Z. Phys. B 1980, 37, 83-87. [CrossRef]

7. Goldman, M.V.; Porkolab, M. Upper hybrid solitons ans oscillating two-stream instabilities. Phys. Fluids 1976, 19, 872-881.

8. Litvak, A.G.; Sergeev, A.M. One dimensional collapse of plasma waves. JETP Lett. 1978, 27, 517-520.

9. Bass, F.G.; Nasanov, N.N. Nonlinear electromagnetic spin waves. Phys. Rep. 1990, 189, 1165-1223. [CrossRef]

10. Makhankov, V.G.; Fedynanin, V.K. Non-linear effects in quasi-one-dimensional models of condensed matter theory. Phys. Rep. 1984, 104, 1-86. [CrossRef]

11. Brihaye, Y.; Hartmann, B.; Zakrzewski, W.J. Spinning solitons of a modified nonlinear Schrödinger equation. Phys. Rev. D 2004, 69, 087701. [CrossRef]

12. Brizhik, L.; Eremko, A.; Piette, B.; Zakrzewski, W.J. Electron self-trapping in a discrete two-dimensional lattice. Physica D 2001, 159, 71-90. [CrossRef]

13. Hartmann, H.; Zakrzewski, W.J. Electrons on hexaonal lattices and applications to nanotubes. Phys. Rev. $B$ 2003, 68, 184-302. [CrossRef]

14. Kurihura, S. Large-amplitude quasi-solitons in superfluid films. J. Phys. Soc. Jpn. 1981, 50, 3262-3267. [CrossRef]

15. Laedke, E.W.; Spatschek, K.H.; Stenflo, L. Evolution theorem for a class of perturbed envelope soliton solutions. J. Math. Phys. 1983, 24, 2764-2769. [CrossRef]

16. Cheng, Y.; Wei, J. Fast and slow decaying solutions for H1-supercritical quasilinear Schrödinger equations. Calc. Var. Part. Differ. Equ. 2019, 58, 144. [CrossRef]

17. Liu, J.; Wang, Y.Q.; Wang, Z.-Q. Soliton solutions for quasilinear Schrödinger equations. II. J. Differ. Equ. 2003, 187, 473-493. [CrossRef]

18. Liu, J.; Wang, Z.-Q. Soliton solutions for quasilinear Schrödinger equations. Proc. Am. Math. Soc. 2003, 131, 441-448. [CrossRef]

19. Poppenberg, M.; Schmitt, K.; Wang, Z.-Q. On the existence of soliton solutions to quasilinear Schrödinger equations. Calc. Var. 2002, 14, 329-344. [CrossRef]

20. Aires, J.F.; Souto, M.A.S. Existence of solutions for a quasilinear Schrödinger equation with vanishing potentials. J. Math. Anal. Appl. 2014, 416, 924-946. [CrossRef]

21. Severo, U.B.; de Carvalho, G.M. Quasilinear Schrödinger equations with unbounded or decaying potentials. Math. Nachr. 2018, 291, 492-517. [CrossRef]

22. Zhang, J.; Tang, X.; Zhang, W. Existence of infinitely many solutions for a quasilinear elliptic equation. Appl. Math. Lett. 2014, 37, 131-135. [CrossRef]

23. Chen, J.; Huang, X.; Cheng, B.; Zhu, C. Some results on standing wave solutions for a class of quasilinear Schrödinger equations. J. Math. Phys. 2019, 60, 091506. [CrossRef]

24. Xue, Y.; Lv, Y.; Tang, C. Existence and nonexistence results for quasilinear Schrödinger equations with a general nonlinear term. Ann. Polon. Math. 2017, 120, 271-293. [CrossRef]

25. Bae, S.; Byeon, J. Standing Waves of nonlinear Schrödinger equations with optimal conditions for potential and nonlinearity. Commun. Pure Appl. Anal. 2013, 12, 831-850. [CrossRef]

26. Gilbarg, D.; Trudinger, N.S. Elliptic Partial Differential Equations of Second Order; Springer: Berlin, Gernmany, 1983.

(C) 2020 by the author. Licensee MDPI, Basel, Switzerland. This article is an open access article distributed under the terms and conditions of the Creative Commons Attribution (CC BY) license (http:/ / creativecommons.org/licenses/by/4.0/). 